RAIRO-Inf. Theor. Appl. 40 (2006) 389-403

DOI: $10.1051 /$ ita:2006015

\title{
A DISTRIBUTED VOTING SCHEME TO MAXIMIZE PREFERENCES *
}

\author{
Peter Auer $^{1}$ And Nicolò Cesa-Bianchi ${ }^{2}$
}

\begin{abstract}
We study the problem of designing a distributed voting scheme for electing a candidate that maximizes the preferences of a set of agents. We assume the preference of agent $i$ for candidate $j$ is a real number $x_{i, j}$, and we do not make any assumptions on the mechanism generating these preferences. We show simple randomized voting schemes guaranteeing the election of a candidate whose expected total preference is nearly the highest among all candidates. The algorithms we consider are designed so that each agent has to disclose only a few bits of information from his preference table. Finally, in the important special case in which each agent is forced to vote for at most one candidate we show that our voting scheme is essentially optimal.
\end{abstract}

Mathematics Subject Classification. 68W15, $91 \mathrm{~B} 12$.

\section{INTRODUCTION}

We consider the problem of choosing a candidate that maximizes the preferences of a set of agents. We assume that, without any prior exchange of information, the agents elect one of $K$ possible candidates via a plurality vote in which each agent may be allowed to vote for more than one candidate. The preference of agent $i$ for candidate $j$ is represented by a number $x_{i, j} \in[0,1]$. We restrict our investigation to distributed, or independent, voting schemes: an agent $i$ has no information

\footnotetext{
* A preliminary version with title "Tight Bounds on the Cumulative Profit of Distributed Voters" appeared in the Proceedings of the 15th ACM Symposium on Principles of Distributed Computing. The authors gratefully acknowledge support by the IST Programme of the European Community, under the PASCAL Network of Excellence, IST-2002-506778. Part of this work was also supported by the Austrian Science Fund FWF (S9104-N04).

1 Dept. of Mathematics and Information Technologies, University of Leoben, Austria.

2 Dipartimento di Scienze dell'Informazione, Università degli Studi di Milano, Italy; cesa-bianchi@dsi.unimi.it
}

(c) EDP Sciences 2006 
about the preferences of other agents, except for the fact that all preferences must lie in the unit interval $[0,1]$. The overall goal is to elect a candidate $j$ maximizing the total preference $G_{j}=x_{1, j}+\cdots+x_{N, j}$, where $N$ is the number of agents.

Our basic voting scheme, LVs (Linear Voting Scheme), is uniform and randomized. All agents use the same voting rule with independent randomizations. The LVS rule is very simple: each agent $i \in\{1, \ldots, N\}$ casts a vote for each candidate $j \in\{1, \ldots, K\}$ with probability $\lambda x_{i, j}$. As the $N \times K$ random choices are made independently, each agent can vote for more than one candidate. We assume that the same parameter $0<\lambda \leq 1$ is used by all agents. To stress the dependence on $\lambda$ we write $\operatorname{LVS}(\lambda)$ to indicate that each agent is running LVS with parameter $\lambda$.

To take into account the important special case when an agent is forced to vote for at most one candidate, we treat the choice $\lambda=1 / K$ separately. When running $\operatorname{LVS}(1 / K)$, each agent $i$ casts a single vote, and the vote is cast for candidate $j$ with probability $x_{i, j} / K$. As $0 \leq x_{i, j} \leq 1$, there might be some remaining probability $1-\frac{1}{K} \sum_{j=1}^{K} x_{i, j}$ which is assigned to the event that agent $i$ abstains. Although the sample space associated to $\operatorname{LVS}(1 / K)$ is different from the sample space associated to $\operatorname{LVS}(\lambda)$ for the other choices of $\lambda$, in Section 2 we provide a simple unified analysis that covers all cases.

A more restricted version of this problem was introduced and studied in [2]. In the follow-up paper [1] a preliminary analysis of $\operatorname{LVs}(1 / K)$ was given. This work extends both these papers and provides a substantially more complete analysis of the general rule $\operatorname{LVS}(\lambda)$.

Our election problem differs from those commonly analyzed in social choice theory, where the preferences of each agent are expressed in terms of a ranking of the candidates. This latter problem, known as rank aggregation, finds its root in Condorcet's voting theory (1785) and arises in disparate fields, including machine learning (collaborative filtering and meta-search) and database middleware (combining results from multiple databases), see the paper by Dwork et al. [7] for a review of existing results and for an application to the reduction of spam influence on web search engines. Note that our analysis also differs from the standard approach in distributed computing (see, e.g., the papers by Papadimitriou et al. $[6,9,10]$ on distributed decision-making with incomplete information): instead of studying how information can be exchanged in order to efficiently make an optimal decision we look at how good a decision can be made despite the fact that information is kept distributed.

In our analysis of voting schemes we seek bounds on the "regret" $G_{\text {best }}-\mathbb{E}\left[G_{A}\right]$ where $G_{\text {best }}=\max _{j} G_{j}$ is the total preference of any optimal candidate and $\mathbb{E}\left[G_{A}\right]$ is the expected total preference achieved by a voting scheme $A$. As we make no assumptions on how the preferences are generated, we are interested in bounds that hold for any assignments of preferences to candidates. In Theorem 2.2 we show that the regret of $\operatorname{LVS}(\lambda)$ is at most $4 \sqrt{\left(G_{\text {best }} / \lambda\right) \ln K}$ for any $\lambda \in(0,1]$. This implies that the regret of $\operatorname{LVS}(1 / K)$, where each agent casts at most one vote, is upper bounded by $4 \sqrt{G_{\text {best }} K \ln K}$. Theorem 3.2 matches this upper bound (up 
to constants), thus showing that the regret of $\operatorname{LVs}(1 / K)$ is $\Theta\left(\sqrt{G_{\text {best }} K \ln K}\right)$. The most complex part of our analysis is the proof of a general lower bound (Th. 3.3) showing that the regret of any (possibly nonuniform) voting strategy, casting at most one vote per agent, is $\Omega\left(\sqrt{K G_{\text {best }}}\right)$.

A scenario where our election naturally arises is when agents want to keep secret their preferences (see, for example, Ephrati and Rosenschein [8]). The above results for $\operatorname{LVS}(\lambda)$ guarantee different degrees of approximation of the optimum $G_{\text {best }}$ as a function of the amount of information, from $\Theta(\ln K)$ to $\Theta(K \ln K)$ bits, revealed by each agent.

Extensions of our linear voting scheme have been used by Chang [3,4] to approximately solve Markov decision processes and for the approximate solution of multiobjective stochastic optimization problems.

Finally, we remark that all of our bounds hold also in the case where each agent only knows unbiased estimates of its preferences.

\section{Notation}

Let $K \geq 1$ be the number of candidates and $N \geq 1$ the number of agents. Let $x_{i, j}$ be the preference of agent $i$ for candidate $j$. Throughout the paper, we assume $x_{i, j} \in[0,1]$ for all $1 \leq i \leq N$ and $1 \leq j \leq K$. The generalization to preferences $x_{i, j} \in[a, b]$ for arbitrary $a<b$ is straightforward by appropriate rescaling.

Each agent $i$, based only on its preference table $\left(x_{i, 1}, \ldots, x_{i, K}\right)$, can vote for one or more canditates or withdraw from the election. The candidate obtaining the largest number of votes gets elected, and ties are broken arbitrarily. The overall goal of the election is to choose some candidate $j \in\{1, \ldots, K\}$ whose total preference $G_{j}=\sum_{i=1}^{N} x_{i, j}$ is as close as possible to the maximum $G_{\text {best }}=$ $\max _{1 \leq j \leq K} G_{j}$. For each voting algorithm $A$, let $G_{A}$ be the total preference of the candidate that gets elected when all agents use algorithm $A$ (observe that $G_{A}$ is a random variable depending on the random choices of the agents).

We define the set $\left\{V_{i, j}: 1 \leq i \leq N, 1 \leq j \leq K\right\}$ of $\{0,1\}$-valued random variables such that $V_{i, j}=1$ if and only if $i$ has voted for $j$. Finally, for each candidate $j \in\{1, \ldots, K\}$, let $V_{j}=\sum_{i=1}^{N} V_{i, j}$ be the number of votes received.

\section{AnAlysis of THE LVS VOting SCHEME}

We use a standard result (see e.g. [11], p. 193) on the sum of independent random variables to bound the probability that candidate $j$ gets too many votes when $G_{\text {best }}-G_{j}$ is large.

Lemma 2.1 (Bernstein's inequality). Let $X_{1}, \ldots, X_{N}$ be independent random variables with $\mathbb{E}\left[X_{i}\right]=0$ and $\left|X_{i}\right| \leq M$ for $i=1, \ldots, N$. If $\sigma^{2} \geq \sum_{i=1}^{N} \operatorname{VAR}\left[X_{i}\right]$ 
then for all $\eta>0$

$$
\mathbb{P}\left\{\sum_{i=1}^{N} X_{i} \geq \eta\right\} \leq \exp \left(-\frac{\eta^{2} / 2}{\sigma^{2}+M \eta / 3}\right)
$$

We now state and prove an upper bound of the regret of $\operatorname{Lvs}(\lambda)$.

Theorem 2.2. For all $\lambda \in(0,1], N \geq 1$, and $K>1$

$$
G_{\text {best }}-\mathbb{E}\left[G_{\text {LVS }(\lambda)}\right] \leq 4 \sqrt{\left(G_{\text {best }} / \lambda\right) \ln K}
$$

Proof. As noted in the introduction, the sample space associated to the randomization of an agent running $\operatorname{LVS}(1 / K)$ is different from the sample space associated to $\operatorname{LVS}(\lambda)$ for $\lambda \neq 1 / K$. This is not a problem, since in our analysis the characterizing property of a voting strategy are the expectations $\mathbb{E}\left[V_{i, j}\right]$, which equal to $\lambda x_{i, j}$ for any choice of $\lambda \in(0,1]$ including $\lambda=1 / K$.

Let $\Delta_{j}=G_{\text {best }}-G_{j}$. Let $\ell \in\{1, \ldots, K\}$ be some optimal candidate such that $G_{\ell}=G_{\text {best }}$ and let $V_{\text {best }}=V_{\ell}$. Note that, for all $j$,

$$
\mathbb{E}\left[V_{\text {best }}-V_{j}\right]=\sum_{i=1}^{N} \lambda\left(x_{i, \ell}-x_{i, j}\right)=\lambda\left(G_{\text {best }}-G_{j}\right)=\lambda \Delta_{j}
$$

and

$\sum_{i=1}^{N} \operatorname{VAR}\left[V_{i, \ell}-V_{i, j}\right] \leq \sum_{i=1}^{N} \mathbb{E}\left[\left(V_{i, \ell}-V_{i, j}\right)^{2}\right] \leq \sum_{i=1}^{N}\left(\mathbb{E}\left[V_{i, \ell}\right]+\mathbb{E}\left[V_{i, j}\right]\right)=\lambda\left(2 G_{\text {best }}-\Delta_{j}\right)$

Define $X_{i, j}=\mathbb{E}\left[V_{i, \ell}-V_{i, j}\right]-\left(V_{i, \ell}-V_{i, j}\right)$. As each voter casts ballots independently, for each $j=1, \ldots, K$ the random variables $X_{1, j}, \ldots, X_{N, j}$ are independent. Furthermore, $\left|X_{i, j}\right| \leq 2, \mathbb{E}\left[X_{i, j}\right]=0$, and $\sum_{i=1}^{N} \operatorname{VAR}\left[X_{i, j}\right]=\sum_{i=1}^{N} \operatorname{VAR}\left[V_{i, \ell}-V_{i, j}\right] \leq$ $\lambda\left(2 G_{\text {best }}-\Delta_{j}\right)$. For any $\alpha>0$ we get

$$
G_{\text {best }}-\mathbb{E}\left[G_{A}\right] \leq \alpha \mathbb{P}\left\{G_{\text {best }}-G_{A} \leq \alpha\right\}+\sum_{j: \Delta_{j}>\alpha} \Delta_{j} \mathbb{P}\left\{V_{j} \geq V_{\ell}\right\}
$$


We upper bound the first term of (1) by $\alpha$. For the second term we find that

$$
\begin{aligned}
\sum_{j: \Delta_{j}>\alpha} \Delta_{j} \mathbb{P}\left\{V_{j} \geq V_{\ell}\right\} & =\sum_{j: \Delta_{j}>\alpha} \Delta_{j} \mathbb{P}\left\{\mathbb{E}\left[V_{\ell}-V_{j}\right]-\left(V_{\ell}-V_{j}\right) \geq \mathbb{E}\left[V_{\ell}-V_{j}\right]\right\} \\
& =\sum_{j: \Delta_{j}>\alpha} \Delta_{j} \mathbb{P}\left\{\sum_{i=1}^{N} X_{i, j} \geq \lambda \Delta_{j}\right\} \\
& \leq \sum_{j: \Delta_{j}>\alpha} \Delta_{j} \exp \left(-\frac{\lambda^{2} \Delta_{j}^{2} / 2}{\lambda\left(2 G_{\text {best }}-\Delta_{j}\right)+(2 / 3) \lambda \Delta_{j}}\right) \\
& \leq \sum_{j: \Delta_{j}>\alpha} \Delta_{j} \exp \left(-\frac{\lambda \Delta_{j}^{2}}{4 G_{\text {best }}}\right) \\
& \leq \alpha
\end{aligned}
$$

for $\alpha=2 \sqrt{\left(G_{\text {best }} / \lambda\right) \ln K}$. Inequality (2) is obtained applying Bernstein's inequality to the sums $\sum_{i=1}^{N} X_{i, j}$ for each $j$. Inequality (3) is proven by observing that $\Delta \mathrm{e}^{-\lambda \Delta^{2} /\left(4 G_{\text {best }}\right)}$ is decreasing in $\Delta$ for $\Delta>\alpha$ and equal to $\alpha / K$ for $\Delta=\alpha$. Thus $G_{\text {best }}-\mathbb{E}\left[G_{A}\right] \leq 2 \alpha$.

In the special cases $\operatorname{LVS}(1 / K)$ and $\operatorname{LVS}(1)$, Theorem 2.2 immediately gives the following bounds:

$$
\begin{aligned}
G_{\text {best }}-\mathbb{E}\left[G_{\mathrm{LVS}(1 / K)}\right] & \leq 4 \sqrt{G_{\text {best }} K \ln K} \\
G_{\text {best }}-\mathbb{E}\left[G_{\mathrm{LVS}(1)}\right] & \leq 4 \sqrt{G_{\text {best }} \ln K} .
\end{aligned}
$$

Note that Theorem 2.2 can be generalized (with minor modifications in the proof) to the case where each voter $i$ does not know the exact value $x_{i, j}$ of the preference assigned to each candidate $j$ but only an unbiased estimate of this value.

\section{Optimality of $\operatorname{LVS}(1 / K)$}

A single-vote strategy is a voting strategy in which each agent can either cast a single vote or abstain. In this section we show that $\operatorname{Lvs}(1 / K)$ is essentially the best possible single-vote strategy. We start by exhibiting a particular set of preference tables on which $\operatorname{LvS}(1 / K)$ incurs regret $\Omega(\sqrt{N K \ln K})$. Combining this with Theorem 2.2 we have that the regret of $\operatorname{LvS}(1 / K)$ is $\Theta(\sqrt{N K \ln K})$. We conjecture that the regret of any single-vote strategy $A$ is at least $\Omega(\sqrt{N K \ln K})$. Although we are not able to prove this by a formal argument, in Section 3.2 we give a proof of a slightly weaker (but very general) lower bound $\Omega(\sqrt{N K})$ that holds for any, even nonuniform, single-vote strategy. 
In the proofs we use the Berry-Esseen theorem [5], Theorem 3, Chapter 9.

Theorem 3.1 (Berry-Esseen). Let $X_{1}, X_{2}, \ldots$ be a sequence of independent random variables with zero mean and finite variance. Let $s_{n}^{2}=\sum_{i=1}^{n} \operatorname{VAR}\left[X_{i}\right]$. If $\mathbb{E}\left[\left|X_{i}\right|^{3}\right] \leq \rho \operatorname{VAR}\left[X_{i}\right]$ then

$$
\sup _{x \in \mathbb{R}}\left|F_{n}(x)-\Phi(x)\right| \leq \frac{c \rho}{s_{n}}
$$

for some constant $c$, where $F_{n}$ is the distribution function of $\sum_{i=1}^{n} X_{i} / s_{n}$ and $\Phi$ is the standard normal distribution function. For i.i.d. random variables $X_{i}$ we have $c \leq 3$, for nonuniform $X_{i}$ we have $c \leq 6$.

\subsection{A LOWER BOUND ON THE REGRET OF ALGORITHM LVS}

We now prove that the bound of Theorem 2.2 is a quite tight estimate of the worst-case regret for $\operatorname{LVS}(1 / K)$.

Theorem 3.2. For all $K \geq 99$ and $N \geq 20 K^{2}$ there are vectors $\boldsymbol{x}_{1}, \ldots, \boldsymbol{x}_{N}$ of preferences such that

$$
G_{\text {best }}-\mathbb{E}\left[G_{\mathrm{LVS}(1 / K)}\right] \geq 0.17 \sqrt{N K \ln K}
$$

Proof. Let $K=2 k-1$ and define the preference vectors

$$
\boldsymbol{x}=\underbrace{1, \ldots, 1}_{k}, \overbrace{0, \ldots, 0}^{k-1} \quad \text { and } \quad \boldsymbol{y}=\underbrace{0, \ldots, 0}_{k-1}, \overbrace{1, \ldots, 1}^{k} \text {. }
$$

We feed preference vector $\boldsymbol{x}$ to $N-n$ voters and preference vector $\boldsymbol{y}$ to the other $n$ voters where $n$, whose precise value will be determined by the analysis, is chosen so that $n \leq N / 2$. Then $G_{\text {best }}=G_{k}=N$ and $G_{j} \leq N-n$ for all $j \neq k$.

Before proceeding with the proof we give a sketch of the main ideas. With the above assignment of preferences, the number of votes for candidates from 1 through $k-1$ is approximately normally distributed with mean $(N-n) / K$ and variance $(N-n) \frac{1}{K}\left(1-\frac{1}{K}\right)$. Thus, among these $k-1$ candidates, the candidate with the maximum number of votes receives about $(N-n) / K+c \sqrt{(N / K) \ln K}$ votes for some constant $c$ with constant probability. On the other hand, candidate $k$ receives about $N / K$ votes. By choosing $n=c \sqrt{(N K) \ln K}$ we find that, with constant probability, a candidate $j \neq k$ receives more votes than candidate $k$. Since the preference for candidate $k$ is at least $n$ plus the preference of any other candidate, this yields $G_{\text {best }}-\mathbb{E}\left[G_{\mathrm{LVS}(1 / K)}\right]=\Omega(\sqrt{N K \ln K})$. 
We start by observing that

$$
\begin{aligned}
G_{\text {best }}- & \mathbb{E}\left[G_{\mathrm{LVS}(1 / K)}\right] \geq n \mathbb{P}\left\{\max _{j \neq k} V_{j}>V_{k}\right\} \\
\geq & n \mathbb{P}\left\{V_{k} \leq N / K, \max _{j \neq k} V_{j}>N / K\right\} \\
= & n\left(\mathbb{P}\left\{V_{k} \leq N / K\right\}-\mathbb{P}\left\{V_{k} \leq N / K, \max _{j \neq k} V_{j} \leq N / K\right\}\right) \\
\geq & n\left(\mathbb{P}\left\{V_{k} \leq N / K\right\}-\mathbb{P}\left\{V_{k} \leq N / K, \max _{1 \leq j \leq k-1} V_{j} \leq N / K\right\}\right) \\
= & n \mathbb{P}\left\{V_{k} \leq N / K\right\} \\
& \times \quad\left[1-\prod_{j=1}^{k-1} \mathbb{P}\left\{V_{j} \leq N / K \mid V_{k} \leq N / K, V_{1} \leq N / K, \ldots, V_{j-1} \leq N / K\right\}\right] \\
\geq & n \mathbb{P}\left\{V_{k} \leq N / K\right\}\left[1-\left(\mathbb{P}\left\{V_{1} \leq N / K\right\}\right)^{k-1}\right] .
\end{aligned}
$$

The last inequality holds because

$$
\mathbb{P}\left\{V_{j} \leq v_{j}\right\} \geq \mathbb{P}\left\{V_{j} \leq v_{j} \mid V_{1} \leq v_{1}, \ldots, V_{t} \leq v_{t}\right\}
$$

for any $t \geq 1$ and $1 \leq j \leq N$, since each voter can vote only for one candidate.

We now apply the Berry-Esseen theorem with $\rho=1$ and obtain

$$
\begin{aligned}
& \mathbb{P}\left\{V_{k} \leq N / K\right\}=\mathbb{P}\left\{\frac{V_{k}-N / K}{\sqrt{\frac{N}{K}\left(1-\frac{1}{K}\right)}} \leq 0\right\} \geq \Phi(0)-\frac{3}{\sqrt{\frac{N}{K}\left(1-\frac{1}{K}\right)}} \\
& \geq \frac{1}{2}-\frac{3}{\sqrt{10 K\left(1-\frac{1}{K}\right)}} \geq \frac{1}{2}-\frac{3}{\sqrt{990}} \geq \frac{2}{5}
\end{aligned}
$$

where we used $N \geq 20 K^{2}$ and $K \geq 99$. Furthermore,

$$
\begin{aligned}
\mathbb{P}\left\{V_{1} \leq N / K\right\} & =\mathbb{P}\left\{\frac{V_{1}-(N-n) / K}{\sqrt{\frac{N-n}{K}\left(1-\frac{1}{K}\right)}} \leq \frac{N / K-(N-n) / K}{\sqrt{\frac{N-n}{K}\left(1-\frac{1}{K}\right)}}\right\} \\
& =\mathbb{P}\left\{\frac{V_{1}-(N-n) / K}{\sqrt{\frac{N-n}{K}\left(1-\frac{1}{K}\right)}} \leq \frac{n}{\sqrt{(N-n)(K-1)}}\right\} \\
& \leq \Phi\left(\frac{n}{\sqrt{(N-n)(K-1)}}\right)+\frac{3}{\sqrt{\frac{N-n}{K}\left(1-\frac{1}{K}\right)}} .
\end{aligned}
$$


Let $n=c \sqrt{N K \ln K}$ where $c$ will be determined in a moment. Then

$$
\frac{n}{\sqrt{(N-n)(K-1)}}=c \sqrt{\frac{N K \ln K}{(N-n)(K-1)}} \leq c \sqrt{2 \frac{99}{98} \ln K}=\sqrt{\frac{\ln K}{2}}
$$

for $c=\sqrt{49 / 198}$. The inequality holds because $N-n \geq \frac{n}{2}$ and $\frac{K}{K-1} \leq \frac{99}{98}$ due to $n \leq \frac{N}{2}$ and $K \geq 99$. Note that, with the above choice of $n$, the condition $n \leq \frac{N}{2}$ is implied by $N \geq K \ln K$, which is in turn implied by our assumption $N \geq 20 K^{2}$.

Using the standard approximation

$$
\Phi(x) \leq 1-\frac{x}{1+x^{2}} \mathrm{e}^{-x^{2} / 2}
$$

we get

$$
\begin{aligned}
\Phi\left(\frac{n}{\sqrt{(N-n)(K-1)}}\right) & \leq \Phi\left(\sqrt{\frac{\ln K}{2}}\right) \\
& \leq 1-\frac{\sqrt{(\ln K) / 2}}{(\ln K) / 2+1} \exp \left(-\frac{1}{4} \ln K\right) \\
& =1-\frac{\sqrt{2 \ln K}}{\ln K+2} K^{-1 / 4} .
\end{aligned}
$$

Moreover, the assumption $N \geq 20 K^{2}$ implies

$$
\frac{3}{\sqrt{\frac{N-n}{K}\left(1-\frac{1}{K}\right)}} \leq \frac{1}{\sqrt{K}}
$$

Thus

$$
\begin{aligned}
\mathbb{P}\left\{\max _{j \neq k} V_{j}>V_{k}\right\} & \geq \frac{2}{5}\left[1-\left(1-\frac{\sqrt{2 \ln K}}{\ln K+2} K^{-1 / 4}+K^{-1 / 2}\right)^{k-1}\right] \\
& =\frac{2}{5}\left[1-\left(1-\frac{\sqrt{2 \ln K}}{\ln K+2} K^{-1 / 4}+K^{-1 / 2}\right)^{\frac{K-1}{2}}\right] \\
& \geq \frac{2}{5}\left[1-\exp \left(-\frac{K-1}{2}\left(\frac{\sqrt{2 \ln K}}{\ln K+2} K^{-1 / 4}-K^{-1 / 2}\right)\right)\right]
\end{aligned}
$$

The argument of $\exp (\cdot)$ is a function decreasing in $K$. The maximum value of the exponential in the range $K \geq 99$ is thus attained at $K=99$ where it takes value not larger than 0.11 . Therefore

$$
\mathbb{P}\left\{\max _{j \neq k} V_{j}>V_{k}\right\} \geq \frac{2}{5}(1-0.11)=0.356 .
$$


Thus we find that

$$
\begin{aligned}
G_{\text {best }}-\mathbb{E}\left[G_{\mathrm{LVS}(1 / K)}\right] & \geq n \mathbb{P}\left\{\max _{j \neq k} V_{j}>V_{k}\right\} \\
& \geq 0.356 \sqrt{\frac{49}{198}} \sqrt{N K \ln K} \\
& >0.17 \sqrt{N K \ln K}
\end{aligned}
$$

concluding the proof.

\subsection{A LOWER BOUND ON THE REGRET OF ANY NONUNIFORM VOTING STRATEGY}

Next, we prove the more general lower bound for arbitrary nonuniform singlevote strategies. The proof is significantly more involved than the proof of the previous lower bound.

Let a nonuniform voting strategy for $N$ voters be a list $\boldsymbol{A}=\left\langle A_{1}, \ldots, A_{N}\right\rangle$ of $N$ voting algorithms, where algorithm $A_{i}$ is run by voter $i=1, \ldots, N$. Throughout this section, we assume that $\boldsymbol{A}$ is single-vote; i.e., each $A_{i}$ either casts a vote for a single candidate or abstains.

Theorem 3.3. Let $K \geq 768$ and $N \geq K^{3} / 64$. Then there is a constant $D>$ $1 / 2500$ such that for any randomized nonuniform voting strategy $\boldsymbol{A}$ there exist preference vectors $\boldsymbol{x}_{1}, \ldots, \boldsymbol{x}_{N} \in\{0,1\}^{K}$ for which

$$
G_{\text {best }}-\mathbb{E}\left[G_{\boldsymbol{A}}\right] \geq D \sqrt{K N}
$$

The proof follows the same idea given in Section 3.1, although some additional issues have to be addressed. A source of difficulty is that we do not make any assumptions on the voting algorithms $A_{1}, \ldots, A_{N}$ (apart from the fact that each algorithm $A_{i}$ should cast at most one vote).

All probabilities considered in the rest of this section are generated by an arbitrary, but fixed, voting strategy $\boldsymbol{A}$.

We will make use of the following lemma, which is a special case of the BerryEsseen theorem.

Lemma 3.4. Let $X_{1}, \ldots, X_{N}$ be a sequence of independent random variables with

$$
X_{n}=\left\{\begin{array}{c}
1 \text { with probability } p_{n}, \\
0 \text { with probability } 1-p_{n}-q_{n}, \\
-1 \text { with probability } q_{n} .
\end{array}\right.
$$

Then the distribution $F$ of the normalized sum

$$
S=\frac{\sum_{n=1}^{N}\left(X_{n}-\mathbb{E}\left[X_{n}\right]\right)}{\sqrt{\sum_{n=1}^{N} \operatorname{VAR}\left[X_{n}\right]}}
$$


satisfies

$$
\sup _{x \in \mathbb{R}}|F(x)-\Phi(x)| \leq \frac{12}{\sqrt{\sum_{n=1}^{N} \operatorname{VAR}\left[X_{n}\right]}}
$$

where $\Phi$ is the normal distribution function.

Proof. To apply the Berry-Esseen theorem we need to find $\rho$ such that $\mathbb{E}\left[\mid X_{n}-\right.$ $\left.\left.\mathbb{E} X_{n}\right|^{3}\right] \leq \rho \operatorname{VAR}\left[X_{n}\right]$. Since $\operatorname{VAR}\left[X_{n}\right]=\mathbb{E}\left[\left(X_{n}-\mathbb{E} X_{n}\right)^{2}\right]$ and $\left|X_{n}-\mathbb{E} X_{n}\right| \leq 2$, we find that $\rho=2$ is sufficient, which gives the lemma.

Let $p_{i, j}(\boldsymbol{x})$ denote the probability that $i$ votes for candidate $j$ when given preference vector $\boldsymbol{x}$ and let $P_{j}(\boldsymbol{x})=\sum_{i=1}^{N} p_{i, j}(\boldsymbol{x})$. Let $G_{\boldsymbol{A}}$ be the random variable denoting $\boldsymbol{A}$ 's total preference. In the following, we assume there are $K=4 k$ candidates. Denote by $\mathcal{X}$ the set of preference vectors $\boldsymbol{x} \in\{0,1\}^{4 k}$ such that $x_{j}=1$ for exactly $k$ indices $j$. Furthermore, denote by $\boldsymbol{e}_{i}$ the preference vector with a single 1 at position $i$.

The next result shows that whenever the difference in the expected number of votes between an arbitrary pair of candidates is high for a given voting strategy, then its regret is large.

Lemma 3.5. For any candidate $j \in\{1, \ldots, 4 k\}$ and for any $\boldsymbol{x} \in \mathcal{X}$ such that $x_{j}=1$, if $N k \geq 1000$ and the voting strategy $\boldsymbol{A}$ is such that $P_{\ell}(\boldsymbol{x}) \geq P_{j}(\boldsymbol{x})+3 \sqrt{N k}$ for some candidate $\ell \neq j$, then there is a choice of preference vectors $\boldsymbol{x}_{1}, \ldots, \boldsymbol{x}_{N}$ for which $G_{\text {best }}-\mathbb{E}\left[G_{\boldsymbol{A}}\right] \geq \sqrt{N k} / 10$.

Proof. Pick $j$ and assume there is $\ell$ such that $P_{\ell}(\boldsymbol{x}) \geq P_{j}(\boldsymbol{x})+3 \sqrt{N k}$. We present a sequence of preference vectors such that $\boldsymbol{A}$ elects a bad candidate with constant probability. We give preference vector $\boldsymbol{x}$ to $N-\sqrt{N k}$ voters and vector $\boldsymbol{e}_{j}$ to the remaining $\sqrt{N k}$ voters, so that $G_{j}=N$ and $G_{h} \leq N-\sqrt{N k}$ for each $h \neq j$. Then $\mathbb{E}\left[V_{\ell}\right] \geq P_{\ell}(\boldsymbol{x})-\sqrt{N k}$ and $\mathbb{E}\left[V_{j}\right] \leq P_{j}(\boldsymbol{x})+\sqrt{N k}$. Hence

$$
\mathbb{E}\left[V_{\ell}-V_{j}\right] \geq P_{\ell}(\boldsymbol{x})-P_{j}(\boldsymbol{x})-2 \sqrt{N k} \geq \sqrt{N k}
$$

Note that $V_{\ell}-V_{j}$ is a sum of independent random variables with values in $\{-1,0,+1\}$. If $\operatorname{VAR}\left[V_{\ell}-V_{j}\right] \geq 900$, we can apply Lemma 3.4 to find that $V_{\ell}>V_{j}$ with probability at least $1 / 2-12 / 30=1 / 10$. Otherwise, by Chebyshev's inequality, we get that $\mathbb{P}\left\{V_{\ell}-V_{j} \leq 0\right\} \leq 900 /(N k) \leq 9 / 10$ for $N k \geq 1000$. Thus the regret is lower bounded by $\left(G_{j}-G_{\ell}\right) \mathbb{P}\left\{V_{\ell}>V_{j}\right\} \geq \sqrt{N k} \mathbb{P}\left\{V_{\ell}>V_{j}\right\} \geq \sqrt{N k} / 10$.

From Lemma 3.5 we get that whenever the same preference vector is fed to all voters, then a good voting strategy must spread its votes evenly.

Corollary 3.6. For any candidate $\ell \in\{1, \ldots, 4 k\}$ and for any preference vector $\boldsymbol{x} \in \mathcal{X}$, if $N k \geq 1000$ and the voting strategy $\boldsymbol{A}$ is such that $P_{\ell}(\boldsymbol{x}) \geq N / k+3 \sqrt{N k}$ then there is a choice of preference vectors $\boldsymbol{x}_{1}, \ldots, \boldsymbol{x}_{N}$ for which $G_{\mathrm{best}}-\mathbb{E}\left[G_{\boldsymbol{A}}\right] \geq$ $\sqrt{N k} / 10$. 
Proof. To apply Lemma 3.5 we have to find some $j$ with $x_{j}=1$ and $P_{j}(\boldsymbol{x}) \leq N / k$. For the purpose of contradiction, assume that for all $j$ with $x_{j}=1, P_{j}(\boldsymbol{x})>N / k$. Then $\sum_{j=1}^{4 k} P_{j}(\boldsymbol{x})>N$, which is not possible ${ }^{1}$.

We are now ready to prove the theorem.

Proof of Theorem 3.3. We divide the $N$ voters into disjoint groups $\mathcal{N}_{1}, \mathcal{N}_{2}, \mathcal{N}_{3}$, and $\mathcal{N}_{4}$. Intuitively, group $\mathcal{N}_{1}$ contains the voters that vote with high probability for candidate 1 or 2 . Group $\mathcal{N}_{2}$ contains the voters whose voting distributions give rise to a high variance in the number of votes for candidates 1 and 2. Group $\mathcal{N}_{3}$ includes all the remaining voters but a small number proportional to $\sqrt{N K}$. Finally, group $\mathcal{N}_{4}$ is used to favor either candidate 1 or candidate 2 . Let the preference vectors $\boldsymbol{x}, \boldsymbol{y}, \boldsymbol{u}, \boldsymbol{v} \in \mathcal{X}$ be chosen as follows

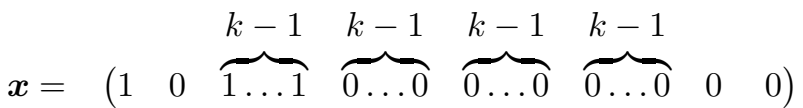

$$
\begin{aligned}
& \boldsymbol{y}=\left(\begin{array}{llllllllll}
1 & 0 & 0 \ldots 0 & 1 \ldots 1 & 0 \ldots 0 & 0 \ldots 0 & 0 & 0
\end{array}\right) \\
& \boldsymbol{u}=\left(\begin{array}{llllllllll}
0 & 1 & 0 \ldots 0 & 0 \ldots 0 & 1 \ldots 1 & 0 \ldots 0 & 0 & 0
\end{array}\right) \\
& \boldsymbol{v}=\left(\begin{array}{llllllllll}
0 & 1 & 0 \ldots 0 & 0 \ldots 0 & 0 \ldots 0 & 1 \ldots 1 & 0 & 0
\end{array}\right) .
\end{aligned}
$$

We use $\mathcal{N}_{g}(\boldsymbol{w})$ to denote the voters in group $\mathcal{N}_{g}$ who receive preference vector $\boldsymbol{w}$ and $V_{i, j}(\boldsymbol{w})$ to denote the random variable $V_{i, j}$ when voter $i$ receives preference vector $\boldsymbol{w}$.

Let $\mathcal{N}_{1}(\boldsymbol{x})$ contain all voters $i$ with $p_{i, 1}(\boldsymbol{x})+p_{i, 2}(\boldsymbol{x}) \geq 1 / 2$. If there are more than $4 N / k+12 \sqrt{N k}$ such voters, then Corollary 3.6 already implies large regret. Thus we may assume the opposite. We add to $\mathcal{N}_{1}(\boldsymbol{x})$ arbitrary voters until $\left|\mathcal{N}_{1}(\boldsymbol{x})\right|=$ N/12.

Let $\mathcal{N}_{1}(\boldsymbol{y})$ contain all voters $i$ not already in $\mathcal{N}_{1}(\boldsymbol{x})$, with $p_{i, 1}(\boldsymbol{y})+p_{i, 2}(\boldsymbol{y}) \geq 1 / 2$. Again by Corollary 3.6, we may assume at most $4 N / k+12 \sqrt{N k}$ such voters. As before, we add arbitrary voters to $\mathcal{N}_{1}(\boldsymbol{y})$ until $\left|\mathcal{N}_{1}(\boldsymbol{y})\right|=N / 12$. Similarly, we define $\mathcal{N}_{1}(\boldsymbol{u})$ and $\mathcal{N}_{1}(\boldsymbol{v})$. We have

$$
p_{i, 1}(\boldsymbol{w})+p_{i, 2}(\boldsymbol{w}) \leq 1 / 2 \quad \text { for each } \boldsymbol{w} \in\{\boldsymbol{x}, \boldsymbol{y}, \boldsymbol{u}, \boldsymbol{v}\} \text { and each } i \notin \mathcal{N}_{1}
$$

For $\mathcal{N}_{2}(\boldsymbol{x})$ we choose those $N / 12$ voters $i$ from the remaining voters with the highest variance $\operatorname{var}\left[V_{i, 1}(\boldsymbol{x})-V_{i, 2}(\boldsymbol{x})\right]$. Analogously, we choose the groups $\mathcal{N}_{2}(\boldsymbol{y})$, $\mathcal{N}_{2}(\boldsymbol{u})$, and $\mathcal{N}_{2}(\boldsymbol{v})$. Let

$$
\sigma^{2}=\sum_{i \in \mathcal{N}_{1} \cup \mathcal{N}_{2}} \operatorname{VAR}\left[V_{i, 1}-V_{i, 2}\right]
$$

\footnotetext{
${ }^{1}$ We believe that this is the only part in the proof where it is crucial to assume that each voter casts just a single vote. Using this assumption, the corollary shows that each candidate receives only about $N / k$ votes. This - in the end - contributes the $\sqrt{K}$ factor in the lower bound of the regret.
} 
By construction of the sets $\mathcal{N}_{2}(\boldsymbol{w})$, we have

$$
\operatorname{VAR}\left[V_{i, 1}(\boldsymbol{w})-V_{i, 2}(\boldsymbol{w})\right] \leq \frac{\sigma^{2}}{N / 12}
$$

for any $\boldsymbol{w} \in\{\boldsymbol{x}, \boldsymbol{y}, \boldsymbol{u}, \boldsymbol{v}\}$ and any voter $i \notin \mathcal{N}_{1} \cup \mathcal{N}_{2}$. Furthermore, $p_{i, 1}(\boldsymbol{w})+$ $p_{i, 2}(\boldsymbol{w}) \leq 1 / 2$ by $(4)$, which implies $\operatorname{VAR}\left[V_{i, 1}(\boldsymbol{w})-V_{i, 2}(\boldsymbol{w})\right] \geq\left(p_{i, 1}(\boldsymbol{w})+p_{i, 2}(\boldsymbol{w})\right) / 2$. Hence,

$$
p_{i, 1}(\boldsymbol{w})+p_{i, 2}(\boldsymbol{w}) \leq 2 \operatorname{VAR}\left[V_{i, 1}(\boldsymbol{w})-V_{i, 2}(\boldsymbol{w})\right] \leq \frac{24 \sigma^{2}}{N} .
$$

The set $\mathcal{N}_{3}$ is chosen arbitrarily and receives preference vectors so that

$$
\left|\mathcal{N}_{3}(\boldsymbol{x})\right|=\left|\mathcal{N}_{3}(\boldsymbol{y})\right|=\left|\mathcal{N}_{3}(\boldsymbol{u})\right|=\left|\mathcal{N}_{3}(\boldsymbol{v})\right|=N / 12-c \sqrt{N k}
$$

where $c$ will be determined by the following analysis. Note that, with respect to the voters in the set $\mathcal{N}_{1} \cup \mathcal{N}_{2} \cup \mathcal{N}_{3}$, candidates 1 and 2 have the same total preference $G$, whereas the total preference of all other candidates is only a constant fraction of $G$. Finally, let $\mathcal{N}_{4}$ denote the remaining $4 c \sqrt{N k}$ voters. The way we assign preference vectors to the voters in $\mathcal{N}_{4}$ will be determined by the following case analysis.

Case 1. $\sigma^{2} \leq C$ for some constant $C>0$ which will be specified later.

For any choice of $\boldsymbol{w}_{i} \in\{\boldsymbol{x}, \boldsymbol{y}, \boldsymbol{u}, \boldsymbol{v}\}$, the probability that none of the voters in $\mathcal{N}_{4}$ votes for 1 or 2 is at least

$$
\begin{aligned}
& \prod_{i \in \mathcal{N}_{4}}\left(1-p_{i, 1}\left(\boldsymbol{w}_{i}\right)-p_{i, 2}\left(\boldsymbol{w}_{i}\right)\right) \\
& \geq \exp \left(-\sum_{i \in \mathcal{N}_{4}}\left[p_{i, 1}\left(\boldsymbol{w}_{i}\right)+p_{i, 2}\left(\boldsymbol{w}_{i}\right)+\left(p_{i, 1}\left(\boldsymbol{w}_{i}\right)+p_{i, 2}\left(\boldsymbol{w}_{i}\right)\right)^{2}\right]\right) \\
& \geq \exp \left(-4 c \sqrt{N k} \frac{24 \sigma^{2}}{N}\left(1+\frac{24 \sigma^{2}}{N}\right)\right) \\
& \geq \exp \left(-96 c C \sqrt{\frac{k}{N}}\left(1+\frac{24 C}{N}\right)\right)
\end{aligned}
$$

where we used the inequality $1-x \geq e^{-x-x^{2}}$ that holds for all $0 \leq x \leq 1 / 2$. Assume that

$$
\mathbb{P}\left\{\sum_{i \notin \mathcal{N}_{4}}\left(V_{i, 1}-V_{i, 2}\right)>0\right\} \geq \frac{1}{3}
$$

That is, with probability at least $1 / 3$, candidate 1 receives, from all the voters not in $\mathcal{N}_{4}$, more votes than candidate 2 . Then

$$
\mathbb{P}\left\{V_{1}>V_{2}\right\} \geq \frac{1}{3} \exp \left(-96 c C \sqrt{\frac{k}{N}}\left(1+\frac{24 C}{N}\right)\right)
$$


for any choice of preference vectors for the voters in $\mathcal{N}_{4}$. Giving each preference vector $\boldsymbol{u}$ and $\boldsymbol{v}$ to half of the voters in $\mathcal{N}_{4}$, we get $G_{j} \leq G_{1} \leq G_{2}-4 c \sqrt{N k}$ for all $j$. Hence the regret is at least

$$
\frac{4 c}{3} \sqrt{N k} \exp \left(-96 c C \sqrt{\frac{k}{N}}\left(1+\frac{24 C}{N}\right)\right) .
$$

The same argument applies if $\mathbb{P}\left\{\sum_{i \notin \mathcal{N}_{4}}\left(V_{i, 2}-V_{i, 1}\right)>0\right\} \geq \frac{1}{3}$.

If $\mathbb{P}\left\{\sum_{i \notin \mathcal{N}_{4}}\left(V_{i, 1}-V_{i, 2}\right)=0\right\} \geq \frac{1}{3}$, then we break the tie in favor of one of the two candidates and apply again the same argument.

Case 2. $\sigma^{2} \geq C$.

We assume that

$$
\sum_{i \notin \mathcal{N}_{4}} \mathbb{E}\left[V_{i, 1}-V_{i, 2}\right] \geq 0 .
$$

(For $\sum_{i \notin \mathcal{N}_{4}} \mathbb{E}\left[V_{i, 1}-V_{i, 2}\right]<0$ the proof proceeds analogously.)

We give each preference vector $\boldsymbol{u}$ and $\boldsymbol{v}$ to half of the voters in $\mathcal{N}_{4}$. By (5) we get that

$$
\mathbb{E}\left[V_{1}-V_{2}\right] \geq \sum_{i \in \mathcal{N}_{4}} \mathbb{E}\left[V_{i, 1}-V_{i, 2}\right] \geq-4 c \sqrt{N k} \frac{24 \sigma^{2}}{N}=-96 c \sigma^{2} \sqrt{\frac{k}{N}}
$$

Furthermore,

Set

$$
\operatorname{VAR}\left[V_{2}-V_{1}\right] \geq \sum_{i \in \mathcal{N}_{1} \cup \mathcal{N}_{2}} \operatorname{VAR}\left[V_{i, 2}-V_{i, 1}\right]=\sigma^{2}
$$

and $a=\mathbb{E}\left[V_{1}-V_{2}\right] / \sigma$. Then

$$
F(x)=\mathbb{P}\left\{\frac{V_{2}-V_{1}-\mathbb{E}\left[V_{2}-V_{1}\right]}{\sqrt{\operatorname{VAR}\left[V_{2}-V_{1}\right]}} \leq x\right\}
$$

$$
\begin{aligned}
\frac{12}{\sqrt{C}} \geq \frac{12}{\sigma} & \geq \frac{12}{\sqrt{\operatorname{VAR}\left[V_{2}-V_{1}\right]}} \quad \text { by }(6) \\
& \geq \Phi(a)-F(a) \quad \text { by Lemma } 3.4 \\
& =\Phi(a)-F\left(-\frac{\mathbb{E}\left[V_{2}-V_{1}\right]}{\sigma}\right) \\
& \geq \Phi(a)-F\left(-\frac{\mathbb{E}\left[V_{2}-V_{1}\right]}{\sqrt{\operatorname{VAR}\left[V_{2}-V_{1}\right]}}\right) \quad \text { by }(6) \\
& =\Phi(a)-\mathbb{P}\left\{\frac{V_{2}-V_{1}-\mathbb{E}\left[V_{2}-V_{1}\right]}{\sqrt{\operatorname{VAR}\left[V_{2}-V_{1}\right]}} \leq-\frac{\mathbb{E}\left[V_{2}-V_{1}\right]}{\sqrt{\operatorname{VAR}\left[V_{2}-V_{1}\right]}}\right\} \\
& =\Phi(a)-\mathbb{P}\left\{V_{1}-V_{2} \geq 0\right\} \\
& \geq \Phi(-96 c \sigma \sqrt{k / N})-\mathbb{P}\left\{V_{1}-V_{2} \geq 0\right\} \quad \text { by }(6) .
\end{aligned}
$$


Hence, $\mathbb{P}\left\{V_{1} \geq V_{2}\right\} \geq \Phi(-96 c \sigma \sqrt{k / N})-12 / \sqrt{C}$. If $\sum_{i=1}^{N}\left(p_{i, 1}+p_{i, 2}\right)>2 \frac{N}{k}+$ $6 \sqrt{N k}$, then Corollary 3.6 implies large regret. Thus, assuming the opposite and using $N \geq k^{3}$, we have that

$$
\sigma^{2} \leq \operatorname{VAR}\left[V_{1}-V_{2}\right] \leq \sum_{i=1}^{N}\left(p_{i, 1}+p_{i, 2}\right) \leq 2 \frac{N}{k}+6 \sqrt{N k} \leq 8 \frac{N}{k}
$$

implying $\sigma \sqrt{k / N} \leq \sqrt{8}$. Thus the regret is at least

$$
4 c \sqrt{N k}\left[\Phi(-96 \sqrt{8} c)-\frac{12}{\sqrt{C}}\right] \text {. }
$$

Choosing $c=1 / 500$ and $C=2000$ we get a regret of at least $\sqrt{K N} / 2500$ in both cases.

\section{Conclusions}

In this paper we have investigated randomized voting schemes for electing a candidate that maximizes the total preference expressed by a set of agents. We have proven a general upper bound on the regret of the linear voting scheme $\operatorname{Lvs}(\lambda)$ which holds for any assignment of preferences. For the special case of single-vote schemes, in which each agent casts at most one vote, we have proven a matching lower bound for the regret of $\operatorname{LVs}(1 / K)$, where $K$ is the number of candidates. Finally, we have shown that $\operatorname{LVS}(1 / K)$ is optimal (up to logarithmic factors) with respect to the class of (possibly nonuniform) randomized single-vote strategies.

A problem left open by our research is the derivation of general lower bounds on the regret of voting strategies where each agent is allowed to vote for more than one candidate. We conjecture that our lower bound techniques for single-vote schemes could be extended to analyze that case as well. A second open problem concerns the strenghtening of the general lower bound on the nonuniform singlevote strategies (Th. 3.3) so to match, up to constant factors, the upper bound proven for $\operatorname{LVs}(1 / K)$. This result also appears to be within reach of our current analytical techniques.

\section{REFERENCES}

[1] P. Auer, P. Caianiello and N. Cesa-Bianchi, Tight bounds on the cumulative profit of distributed voters, in Proceedings of the 15th ACM Symposium on Principles of Distributed Computing. ACM Press (1996) 312.

[2] P. Caianiello, P. Crescenzi and A. Marchetti-Spaccamela, Distributed voting and maximum satisfiability. Unpublished manuscript (1993).

[3] H.S. Chang, Multi-policy iteration with a distributed voting. Math. Methods Oper. Res. 60 (2004) 299-310.

[4] H.S. Chang, On the probability of correct selection by distributed voting in stochastic optimization. J. Optim. Theory Appl. 125 (2005) 231-240. 
[5] Y.S. Chow and H. Teicher, Probability Theory. Springer (1988).

[6] X. Deng and C.H. Papadimitriou, Distributed decision-making with incomplete information, in Proceedings of the 12ft IFIP Congress. Madrid (1992).

[7] C. Dwork, R. Kumar, M. Naor and D. Sivakumar, Rank aggregation revisited, in Proceedings of the 10th International World Wide Web Conference (2001) 96-104.

[8] E. Ephrati and J.S. Rosenschein, Reaching agreement through partial revelation of preferences, in Proceedings of the 10th European Conference on Artificial Intelligence (1992) 229-233.

[9] C.H. Papadimitriou and M. Yannakakis, On the value of information in distributed decision making, in Proceedings of the 10th ACM Symposium on Principles of Distributed Computing. ACM Press (1991) 61-64.

[10] C.H. Papadimitriou and M. Yannakakis, Linear programming without the matrix, in Proceedings of the 25th ACM Symposium on the Theory of Computing. ACM Press (1993) $121-129$.

[11] D. Pollard, Convergence of Stochastic Processes. Springer (1984). 